\title{
Preparation, characterization and antimicrobial assessment of selected ciprofloxacin salts
}

\author{
IMAD I. HAMDAN ${ }^{1}$ \\ DINA EL-SABAWI ${ }^{2}$ \\ RULA DARWISH ${ }^{2}$ \\ LINA A. DAHABIYEH ${ }^{1, *}$ \\ ${ }^{1}$ Department of Pharmaceutical Sciences \\ School of Pharmacy, University of Jordan \\ Amman 11942, Jordan \\ ${ }^{2}$ Department of Pharmaceutics and \\ Pharmaceutical Technology \\ School of Pharmacy, University of Jordan \\ Amman 11942, Jordan
}

Accepted September 18, 2020

Published online October 6, 2020

\begin{abstract}
The formation of salts is considered a simple strategy to modify the physicochemical properties of active pharmaceutical ingredients. In this study, seven novel binary and ternary organic salts of ciprofloxacin $(\mathrm{CP})$ were prepared with benzoic acid (BA), acetylsalicylic acid (ASA), $p$-coumaric acid (PCMA) and $p$-aminosalicylic acid (PASA). They were characterized by spectroscopic techniques and differential scanning calorimetry. Solubility and partition coefficients values were also measured. Evaluation of the antimicrobial activity of the organic salts against Staphylococcus aureus and Staphylococcus epidermidis revealed that most of the new salts had higher antimicrobial activity than CP$\mathrm{HCl}$ against both strains. The most active compounds against S. epidermidis and S. aureus were CP-PASA and CPPCMA, resp., which were up to fourteen times more potent than parent $\mathrm{CP}-\mathrm{HCl}$. Our findings indicated a strong correlation between the lipophilicity of the formed salts and their antimicrobial activity and showed that an optimum value of lipophilicity $(\log P=0.75)$ seemed to be necessary to maximize the antimicrobial activity. These findings highlighted the improved physical, thermal and antimicrobial properties of the new salts of $\mathrm{CP}$ that can aid in providing higher bioavailability than $\mathrm{CP}-\mathrm{HCl}$.
\end{abstract}

Keywords: ciprofloxacin, salts, partition coefficient, Staphylococcus aureus, Staphylococcus epidermidis

Ciprofloxacin (CP, Fig. 1) is a broad-spectrum fluoroquinolone antimicrobial agent that is widely used for treating various infections $(1,2) . \mathrm{CP}$ is effectively soluble at the $\mathrm{pH}$ values of most parts of the gastrointestinal tract (GIT) as it is mainly positively charged [pKa of the basic amino group is 8.8 (3)]. CP exhibits minimum solubility at its isoelectric point ( $\mathrm{pH} 7.4)$ being a zwitterion $(3,4)$. Nevertheless, $\mathrm{CP}$ was reported to have a good oral bioavailability of 60-78 \% (5). Organic anion transporters have been shown to actively transport $\mathrm{CP}$, thus contributing to the observed, otherwise unexpected, high bioavailability (6). Previous reports have shown that concurrent administration of diclofenac sodium (DS) with $\mathrm{CP}$ resulted in a significant increase in its bioavailability (7). Further investigations

\footnotetext{
*Correspondence; e-mail: 1.dahabiyeh@ju.edu.jo
} 
<smiles>O=C(O)c1cn(C2CC2)c2cc(N3CCNCC3)c(F)cc2c1=O</smiles>

Ciprofloxacin $(\mathrm{CP})$<smiles>O=C(O)c1ccccc1</smiles>

Benzoic acid (BA)<smiles>CC(=O)Oc1ccccc1C(=O)O</smiles><smiles>Nc1ccc(C(=O)O)c(O)c1</smiles><smiles>O=C(O)/C=C/c1ccc(O)cc1</smiles>

Acetylsalicylic acid (ASA) $p$-Aminosalicylic acid (PASA) $p$-Coumaric acid (PCMA)

Fig. 1. Chemical structures of ciprofloxacin and the four organic acids used as anion partners.

from our laboratory, revealed that DS forms ion-pair salts with CP. The resulting salts were shown to have modified physicochemical properties, partition coefficient and, to some extent, antimicrobial activity (8). Ternary associates, involving one molecule of CP and two molecules of DS, have also been shown to form, and, interestingly, possess solubilities different than that of $\mathrm{CP}$ or its 1:1 salt. $\mathrm{CP}$ has also been reported to form salts with carboxylic acids with a potential improvement of aqueous solubility $(4,9,10)$. Although salt formation provides a simple strategy with promising potential to favorably modify the pharmaceutical properties of the two entities, the correct choice of the organic conjugate is crucial to provide salts with improved properties.

In this study, we aimed at preparing new salts of $\mathrm{CP}$ with some anionic species. Such salts might in principle improve the antimicrobial performance of $\mathrm{CP}$, particularly in topical and ophthalmic preparations. Topical and ophthalmic drug delivery represent a particular challenge as the drug should strike the delicate balance between hydrophilicity and lipophilicity for the successful transport through the involved tissues (11).

Toward this end, four anionic compounds were particularly chosen with the focus on preparing ion pairs with the cationic CP. The model anionic compounds (Fig. 1) were benzoic acid (BA), acetylsalicylic acid (ASA), $p$-coumaric acid (PCMA) and $p$-aminosalicylic acid (PASA). Interestingly, binary and ternary salts involving $\mathrm{CP}, \mathrm{PCMA}$ and any of the other three anions were formed and exhibited different properties along with promising antimicrobial activities.

\section{EXPERIMENTAL}

\section{Preparation of binary and ternary salts}

$\mathrm{CP}$ (purity $>98 \%$ ), as the hydrochloride salt (CP-HCl), was kindly supplied by Jordan Pharmaceutical Manufacturer (JPM, Jordan) while other compounds were purchased from Sigma-Aldrich (USA) and certified to have purities $>98 \%$. All organic acids were supplied in the free acid form except benzoic acid which was employed as sodium benzoate. 
Ciprofloxacin ( $1.18 \mathrm{~g}, 3.2 \mathrm{mmol})$ was dissolved in $40 \mathrm{~mL}$ of distilled water. An equimolar amount of each among four anion compounds to be tested, benzoic acid (BA), acetylsalicylic acid (ASA), $p$-coumaric acid (PCMA) and $p$-aminosalicylic acid (PASA), was dissolved in the minimum amount of $20 \%(V / V)$ methanol in water. The two solutions were mixed and left in the hood until crystals or a precipitate appeared (2-3 days). The obtained solids were isolated by filtration and were left to dry for 3 days in a $\mathrm{CaCl}_{2}$ desiccator.

\section{Characterization of prepared salts by FTIR, ${ }^{1} \mathrm{H} N \mathrm{~N}$ R and DSC}

Fourier-transform infrared (FTIR) spectra were recorded using Thermo NICOLET NEXUS 670 FTIR spectrometer (ThermoFisher Scientific, USA) with OMNIC software at 4 $\mathrm{cm}^{-1}$ spectral resolution and 32 scans from 4000 to $400 \mathrm{~cm}^{-1}$ using the standard $\mathrm{KBr}$ disc method.

For proton nuclear magnetic resonance $\left({ }^{1} \mathrm{H}\right.$ NMR) analysis of the salts, samples (15 mg) were dissolved in deuterated dimethyl sulfoxide (DMSO, Sigma-Aldrich) and ${ }^{1} \mathrm{H}$ NMR spectra were recorded on a Perkin Elmer 350MHz NMR spectrophotometer (Perkin Elmer, USA). The temperature was kept constant at $308 \mathrm{~K}$.

Differential scanning calorimetry (DSC) analysis was carried out using a Mettler Toledo DSC823e calorimeter (Mettler Toledo, Switzerland) configured to a Mettler_star software system. Samples (4-5 mg) were weighed and scanned in sealed aluminum pans with pierced cover. The instrument was calibrated with indium as a reference. Thermograms were recorded under a dry nitrogen atmosphere $\left(80 \mathrm{~mL} \mathrm{~min}^{-1}\right)$, over a temperature range of $30-300{ }^{\circ} \mathrm{C}$ and at a heating rate of $10^{\circ} \mathrm{C} \mathrm{min}^{-1}$.

Melting points were also determined using standard capillary type melting point apparatus.

\section{Solubility and partition coefficient of the prepared salts}

Solubility and apparent partition coefficient studies were performed in triplicate as previously described (8). Briefly, the excess solid material of CP or any of the prepared salts was added to $4 \mathrm{~mL}$ of phosphate buffer ( $\mathrm{pH}$ 6.8), left on a shaker water bath for $24 \mathrm{~h}$ and then $1 \mathrm{~mL}$ was centrifuged. The supernatant $(100 \mu \mathrm{L})$ was diluted and then injected into high-performance liquid chromatography (HPLC). For the determination of partition coefficient values, $2 \mathrm{~mL}$ of the remaining supernatant solutions were mixed with $2 \mathrm{~mL}$ of octanol for $24 \mathrm{~h}$ on a shaker water bath. Aliquots of the aqueous layer were properly diluted before being injected into HPLC. Results were reported as apparent $\log P$ values.

\section{HPLC analysis}

The previously optimized and validated HPLC method (8) was used to determine the amount of CP. A Merck Hitachi isocratic HPLC pump, equipped with a Merck UV detector (Japan), phenyl $150 \times 4.5 \mathrm{~mm}$ i.d, 5 - $\mu \mathrm{m}$ particle size column (ThermoFisher Scientific, USA) were used, and the mobile phase was composed of acetonitrile and $50 \mathrm{mmol} \mathrm{L}^{-1}$ phosphate buffer ( $\mathrm{pH}$ 6.5) in a volume ratio $25: 75$, with the overall mixture containing $0.2 \%(\mathrm{~m} / \mathrm{V})$ citric acid. The flow rate was maintained at $1.5 \mathrm{~mL} \mathrm{~min}^{-1}$ and the detection wavelength was set at $277 \mathrm{~nm}$. All data were processed using Clarity lite ${ }^{\circledR}$ software. 
I. I. Hamdan et al.: Preparation, characterization and antimicrobial assessment of selected ciprofloxacin salts, Acta Pharm. 71 (2021) 365-382.

\section{Determination of antimicrobial activity}

Standard laboratory strains of Staphylococcus aureus (ATCC 6538) and Staphylococcus epidermidis (ATCC 14990), bought from ATCC (American Type Culture Collection, Manassas, VA, USA), were studied. Stock cultures were maintained at $4{ }^{\circ} \mathrm{C}$ on slopes of nutrient agar. Cultures for experiments were prepared by transferring a sample from the stock cultures into tryptic soy broth and were standardized to match 0.5 McFarlands to an OD at $600 \mathrm{~nm}$ of $0.1( \pm 0.02)$ according to Clinical and Laboratories Standards Institute (CLSI) 2007.

Stock solutions of prepared salts were prepared by dissolving the solid crystalline and powdered material in DMSO to get the concentration of $5 \mathrm{mg} \mathrm{mL}^{-1}$, then they were filtered twice through a $0.22-\mu \mathrm{m}$ syringe filter. After that, compounds were freshly diluted with tryptic soy broth to yield the required concentration. The concentrations of the tested compounds were in the range $200-0.244 \mu \mathrm{g} \mathrm{mL} \mathrm{m}^{-1}$. Literature showed that ciprofloxacin MICs for S. aureus (ATCC 6538) and S. epidermidis (ATCC 14990) were in the range of 0.25 and $0.5 \mu \mathrm{g} \mathrm{mL} \mathrm{m}^{-1}$ and $0.125-0.3 \mu \mathrm{g} \mathrm{mL} \mathrm{L}^{-1}$, resp. $(12,13)$. These values may differ in different labs, thus, the range of $200-0.244 \mu \mathrm{g} \mathrm{mL}^{-1}$ was selected.

The minimum inhibitory concentration (MIC) of the compounds was determined by the NCCLS broth microdilution reference method (14) with slight modification with DMSO as the solvent and tested for antibacterial activity as a negative control. Briefly, MIC tests were performed in 96-flat bottom microtiter plates (TPP, Switzerland). The ELx800UV universal microplate reader (BioTek, USA) was used to determine the turbidity in the wells. Bacterial suspensions were prepared in sterilized distilled water in a concentration around $1 \times 10^{7} \mathrm{CFU} \mathrm{mL} \mathrm{m}^{-1}$ which was standardized according to $0.5 \mathrm{McF}$ arland suspensions as depicted above. MIC was calculated as the average of two successive concentrations of the tested compound showing growth and no growth, recpectively. Control tests for each experiment were performed. A positive culture was done to ensure the growth of the bacteria in the culture media (i.e., the culture media was properly chosen to give accurate results) and thus it is done using the media with the bacteria alone. $\mathrm{CP}-\mathrm{HCl}$ was used as the standard antibacterial agent.

\section{DNA spectroscopic titrations}

A stock solution of each of the tested products was prepared by dissolving $8 \mathrm{mg}$ in 0.5 $\mathrm{mL}$ of DMSO, however, $\mathrm{CP}-\mathrm{HCl}$ was dissolved in distilled water instead of DMSO as it was freely soluble in water and not sufficiently soluble in DMSO. Stock solutions were appropriately diluted (ca 500 times) in phosphate buffer ( $\mathrm{pH}$ 6.8) to give acceptable UV absorbance values (in the range of $0.5-1$ ) when measured at $330 \mathrm{~nm}$. Diluted solutions $(500 \mu \mathrm{L})$ were transferred to a quartz UV micro-cell. Calf thymus DNA (CT-DNA, Sigma-Aldrich) solutions were prepared in Tris buffer ( $\mathrm{pH}$ 7.4). The DNA solution was added directly to the sample cell as well as the reference cell in increments of $50 \mu \mathrm{L}$. Solutions were well mixed inside the cells and left to stand for $10 \mathrm{~min}$ after each addition of the DNA solution. The absorbance of the final solution was measured at $330 \mathrm{~nm}$ using a Cecil UV/Vis spectrophotometer (Cecil Instrumentation Services Ltd, UK). Binding curves were obtained for each substance by plotting the absorbance value against the molar ratio of CP to DNA (in base pairs). Further assessment of the binding process and estimation of the binding constant was based on the procedures adopted by Wolfe et al. (15). 


\section{RESULTS AND DISCUSSION}

\section{Characterization of products}

Overall, seven products were obtained (Table I); some appeared as very fine crystals, while the others were apparently amorphous. Four of the products were binary salts, i.e., assumed to be composed of $\mathrm{CP}$ and either of the four organic acids and thus were labeled as CP-PCMA, CP-ASA, CP-PASA and CP-BA. The remaining three products were assumed to be ternary structures of CP, PCMA and either ASA, PASA or BA. The products were extensively characterized before their antibacterial activity was evaluated as presented in the sections that follow.

Melting points and DSC. - Melting points of different compounds are shown in Table I. Generally, a combination of CP with four among investigated anions significantly lowers the melting point of the formed binary and ternary salts compared to the starting material, CP-hydrochloride $(\mathrm{CP}-\mathrm{HCl})$ as noted in Table I. The salts exhibited melting points within a narrow temperature range which indicates the formation of the hypothesized products of reasonable purity. It is noteworthy that ternary complexes exhibited lower melting points than their binary counterparts with an exception of ASA compounds which exhibited higher melting points when compared to its binary product (Table I). Normally, the change in the melting point for salts depends on the type of the anion used, the shape and the order of the molecules in the salts, and the force that maintain their ordered structure (16). Therefore, it is expected that the binary compounds involve more symmetrical and ordered structures along with higher intermolecular forces between molecules within the molecular lattice than the ternary systems.

DSC thermograms (Fig. 2) were generally in support of melting point data and provided further details regarding the melting behavior of the various products. The case of binary and ternary salts involving ASA and PCMA is discussed in more detail here (Fig.

Table I. Melting points, solubility and log $\mathrm{P}$ values of $\mathrm{CP}-\mathrm{HCl}$ and its different salts

\begin{tabular}{lcccc}
\hline Compound & $\begin{array}{c}\text { Melting point } \\
\left({ }^{\circ} \mathrm{C}\right)\end{array}$ & $\begin{array}{c}\text { Solubility } \\
\left(\mathrm{mg} \mathrm{mL}^{-1}\right)^{\mathrm{a}}\end{array}$ & $\log P^{\mathrm{a}}$ & $\begin{array}{c}\mathrm{CP}(\%) \\
{[\text { molar ratio }]^{\mathrm{b}}}\end{array}$ \\
\hline CP-PASA-PCMA & $159-162$ & 5.4 & 0.204 & $55.3[1: 1: 1]$ \\
CP-ASA-PCMA & $196-199$ & 2.8 & 0.322 & $35.6[1: 1: 2]$ \\
CP-BA-PCMA & $215-217$ & 1.2 & 0.544 & $61.2[1: 1: 1]$ \\
CP-PASA & $206-207$ & 1.5 & 0.721 & $65.1[1: 1]$ \\
CP-ASA & $122-126$ & 1.8 & 0.920 & $24.6[1: 4]$ \\
CP-BA & $234-236$ & 2.0 & 0.863 & $72.3[1: 1]$ \\
CP-PCMA & $224-227$ & 1.5 & 0.716 & $61.9[1: 2]$ \\
CP-HCl & $317-319$ & 27.9 & -0.222 & - \\
\hline
\end{tabular}

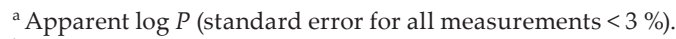

${ }^{\mathrm{b}}$ Determined by HPLC. 

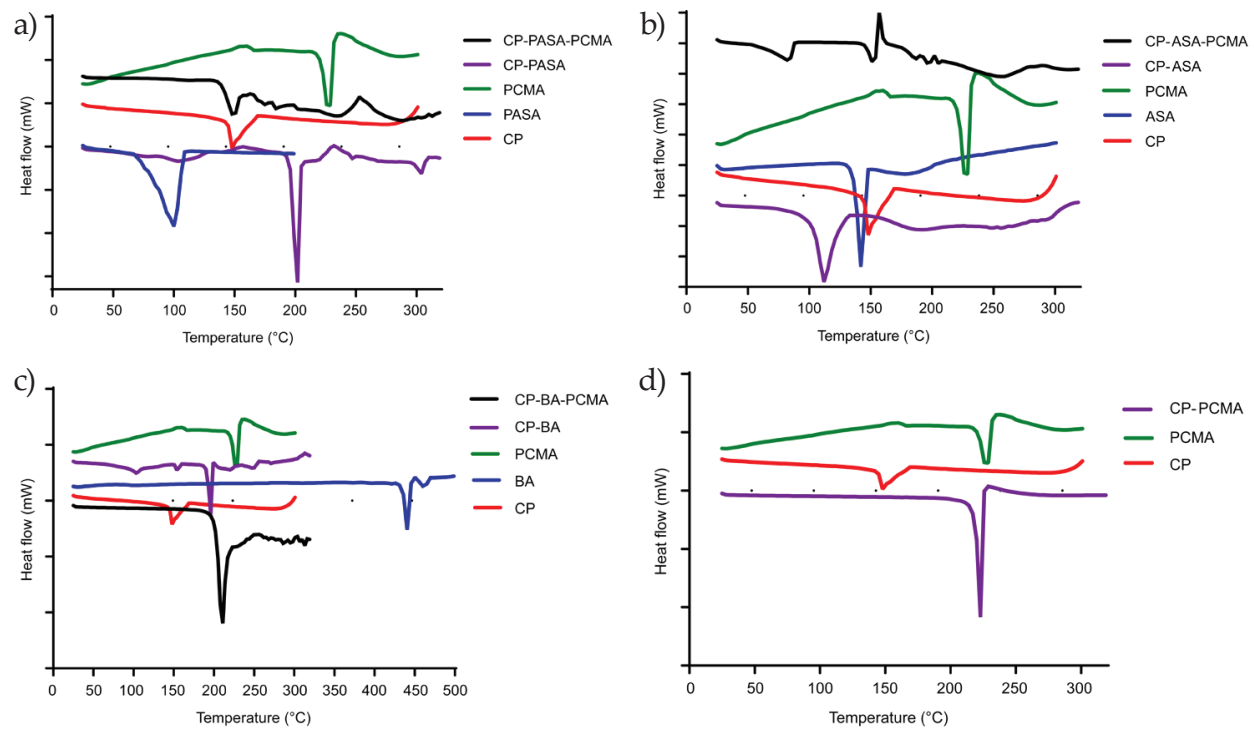

Fig. 2. DSC thermograms of the parent moieties and binary and ternary salts obtained from CP and: a) PASA, b) ASA, c) BA and d) PCMA.

2b). The thermograms in the studied temperature range, for the free parent compounds: $\mathrm{CP}-\mathrm{HCl}, \mathrm{ASA}$, and PCMA showed clear endotherms at about 145,140 and $240{ }^{\circ} \mathrm{C}$ (sharp), resp. However, the binary salt CP-ASA and CP-PCMA showed only sharp peaks at 115 and $225^{\circ} \mathrm{C}$, resp., which might serve as good evidence of the formation of new entities (the salts). For the ternary system (CP-ASA-PCMA), however, the thermogram was showing two different endotherms at about 80 and $145^{\circ} \mathrm{C}$. Additionally, an exothermic peak was observed in the thermogram of the ternary product CP-ASA-PCMA at about $155^{\circ} \mathrm{C}$ which is suggestive of structural rearrangement after initial melting.

The additional exothermic peak (suggestive of molecular rearrangement) was indeed a characteristic feature in the DSC thermograms of the ternary products (Figs. 2a-c). This was consistent with our suggestion that the ternary complexes resulted in less ordered molecular structures compared to binary complexes and, therefore, might undergo molecular rearrangement once in the liquid state (after initial melting).

$\mathrm{CP}$ content, solubility and $\log \mathrm{P}$ values of the prepared salts. - The content of $\mathrm{CP}$ in each of the products was assayed using the HPLC method (8) when CP was very well separated from the employed counterion (organic acid). Although ion-exchange chromatography is the most frequently reported method for the separation of the counterions of organic salts, other chromatographic modes including reversed-phase and mixed phases have also been reported to be successful (17-19).

In this study, the reversed-phase column was found successful in separating $\mathrm{CP}$ from counteranions. This was verified by showing that the counteranions having good UV absorptivity were eluted at a significantly lower retention time than CP. Representative chromatograms showing the separation of $\mathrm{CP}$ from the organic anions are presented in 
a)

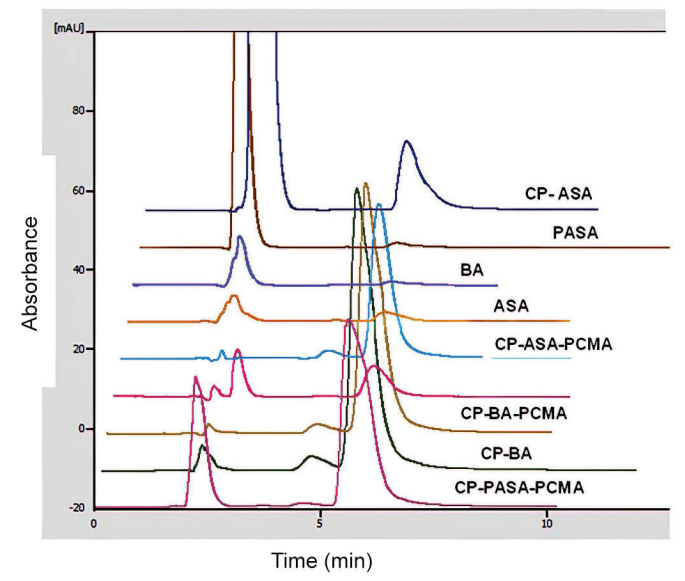

b)

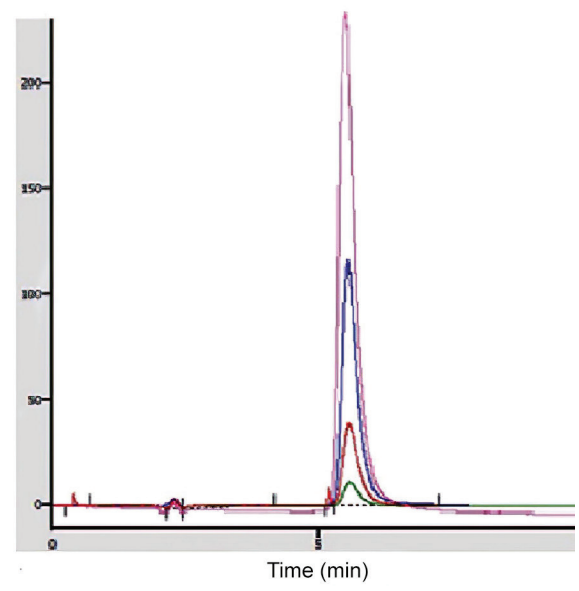

Fig. 3. a) Representative chromatograms of various prepared salts showing the separation of $\mathrm{CP}\left(t_{\mathrm{r}}\right.$ at about $6 \mathrm{~min}$ ) from the other organic anions (early eluting peaks at $2.5 \mathrm{~min}$ ). b) Representative chromatograms of the standard $\mathrm{CP}-\mathrm{HCl}$ employed for the construction of the calibration curve $(2-50 \mu \mathrm{g}$ $\mathrm{mL}^{-1}$ range).

Fig. 3a. The method showed precision with RSD $<2.5 \%$ at different concentration levels, and accuracy within the range $98-103 \%$. Additionally, the HPLC method was linear over the concentration range of $5-50 \mu \mathrm{g} \mathrm{mL} \mathrm{m}^{-1}$ for $\mathrm{CP}$, with $R^{2}>0.998$. Results expressed as a percentage of $\mathrm{CP}$ in the products are presented in Table I. The differences between the experimentally found percentage of $\mathrm{CP}$ and their expected values (i.e., $100 \%$ assuming the product was composed of $\mathrm{CP}$ only) were considered to correspond to the accompanying anionic species. Therefore, by using the molar mass of $\mathrm{CP}$ and that of the different anions employed, it was possible to estimate the likely molar association ratios between $\mathrm{CP}$ and anions that fit the determined percentage of $\mathrm{CP}$ within a margin of error $<4 \%$.

The data in Table I revealed that, as expected, only BA and PASA formed 1:1 salts with CP, while PCMA and ASA associated with CP at ratios of 2:1 and 4:1, resp. When PCMA existed in solution along with other anions, ternary complexes involving PCMA, CP and other anions were formed. All of these ratios (estimates based on HPLC determination of $\mathrm{CP}$ content in the product) were further supported by NMR spectra for the products (vide infra).

Aqueous solubility of most products was significantly lower ( 10 times) than that of the parent $\mathrm{CP}-\mathrm{HCl}$ (Table I). $\log P$ values were also higher for the prepared salts compared to $\mathrm{CP}-\mathrm{HCl}$. The binary CP-ASA salt had the highest $\log P(0.92)$ whereas the ternary $\mathrm{CP}-$ PASA-PCMA salt showed the lowest $\log P(0.204)$. The observed differences in solubility and $\log P$ values between the seven prepared $\mathrm{CP}$ salts and the parent free $\mathrm{CP}-\mathrm{HCl}$ provide evidence of the formation of salts and maintenance of their structures in solution; if the salts did not hold in solution then $\log P$ values would not be expected to change. Our results indicate that the formation of different salts of $\mathrm{CP}$ is clearly affecting its physicochemical properties which depend mainly on the type of the organic anion used. It is 
a)

b)

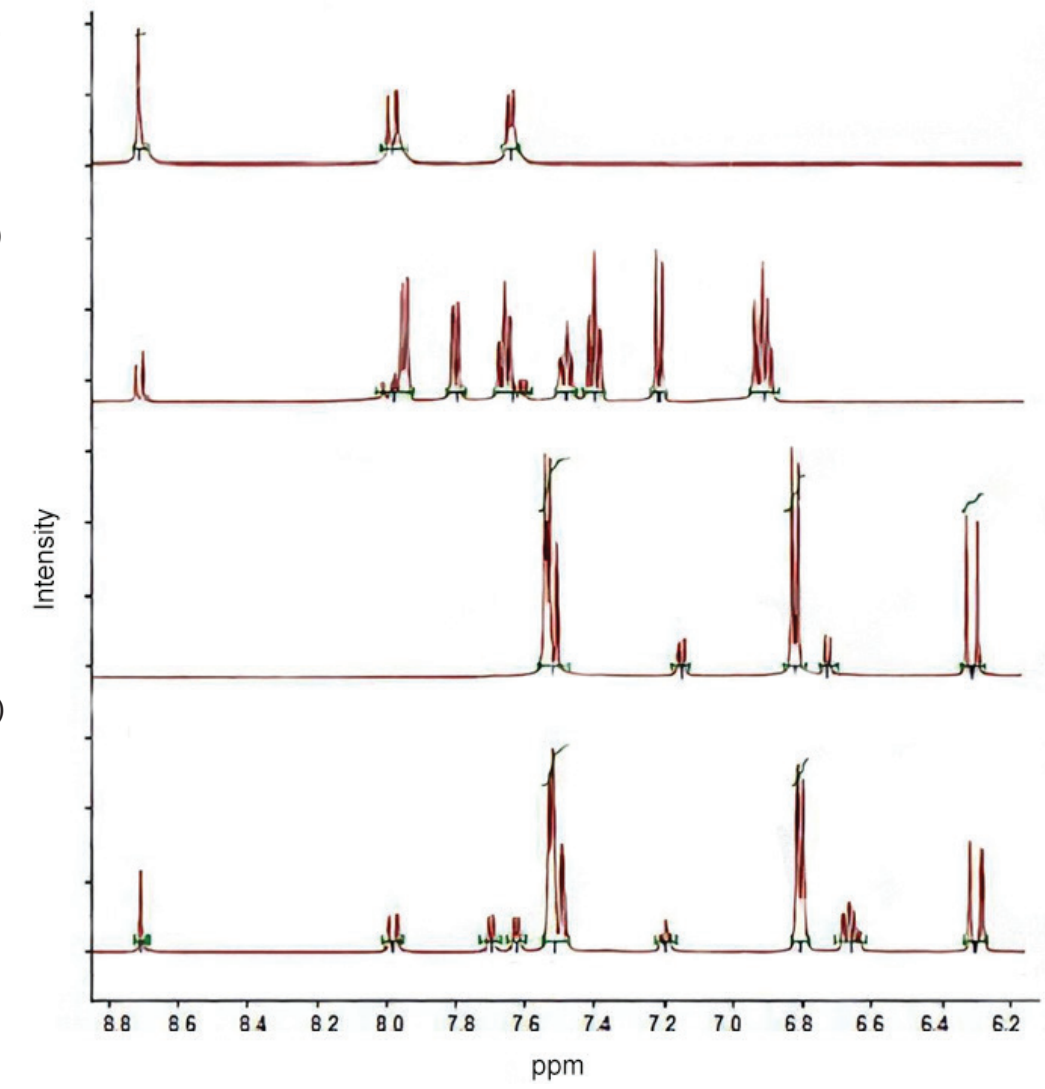

Fig. 4. ${ }^{1} \mathrm{H}$ NMR spectra of: a) CP-HCl, b) CP-ASA, c) CP-PCMA, d) CP-ASA-PCMA.

expected that the noted differences in $\log P$ values among the products might result in different permeation of the drug through bacterial cell wall with a possibility of attaining optimal effects.

${ }^{1} \mathrm{H}$ NMR and FTIR spectroscopy. $-{ }^{1} \mathrm{H}$ NMR spectra were obtained for all products and $\mathrm{CP}-\mathrm{HCl}$ (Fig. S1). A summary of the obtained peaks and their assignments for all products is also presented in Table II. The ${ }^{1} \mathrm{H}$ NMR for the binary and the ternary salts of ASA (as an example) with CP is presented in Fig. 4.

Generally, spectra of the prepared salts accorded well with those previously reported for their parent compounds, $\mathrm{CP}$ and the four organic anions (20). Thus aromatic protons number 2, 5 and 8 in CP (Fig. 1) resonated at 8.69 (singlet), 7.75 (doublet) and 7.64 (doublet) ppm, resp. (Fig. 4a). Assignments of protons on ASA and its related products were based on literature reports on ASA in addition to the characteristic multiplicity pattern for each proton and the relative peak area for each signal (Fig. 4b), e.g., proton No. 5 on ASA 
I. I. Hamdan et al.: Preparation, characterization and antimicrobial assessment of selected ciprofloxacin salts, Acta Pharm. 71 (2021) 365-382.

Table II. Summary of $1 H$ NMR data within the aromatic region for the formed CP products

\begin{tabular}{|c|c|}
\hline Compound & $\begin{array}{l}\text { Chemical shift }\left(\mathrm{cm}^{-1}\right) \text { (multiplicity, number of protons, assigned protons } \\
\text { within the structure) }\end{array}$ \\
\hline Ciprofloxacin- $\mathrm{HCl}$ & 8.69 (s, 1H, no. 2); 7.75 (d, 1H, no. 5); 7.64 (d, 1H, no. 8) \\
\hline \multirow[b]{2}{*}{ CP-ASA } & CP protons: 8.68 (s, $1 \mathrm{H}$, no.2); $7.96(\mathrm{t}, 1 \mathrm{H}$, no. 5$) ; 7.59(\mathrm{~d}, 1 \mathrm{H}$, no. 8$)$ \\
\hline & $\begin{array}{l}\text { ASA protons: } 6.89(\mathrm{q}, 2 \mathrm{H}, \text { no. } 2 \text { and } 4) ; 7.19(\mathrm{~d}, 1 \mathrm{H}, \text { no. } 2) ; 7.38(\mathrm{t}, 1 \mathrm{H}, \text { no. } 4) ; \\
7.45(\mathrm{t}, 1 \mathrm{H}, \text { no. } 3) ; 7.63(\mathrm{t}, 1 \mathrm{H}, \text { no. } 3) ; 7.77(\mathrm{dd}, 1 \mathrm{H}, \text { no. } 5) ; 7.92(\mathrm{dd}, 1 \mathrm{H}, \text { no. } 5)\end{array}$ \\
\hline \multirow[b]{2}{*}{ СР-PCMA } & CP protons: $7.51(\mathrm{~s}, 1 \mathrm{H}$, no. 2$) ; 7.2(\mathrm{~d}, 1 \mathrm{H}$, no. 5); $6.7(\mathrm{~d}, 1 \mathrm{H}$, no. 8$)$ \\
\hline & $\begin{array}{l}\text { PCMA protons: } 7.51(\mathrm{~d}, 2 \mathrm{H}, \text { no. } 2 \text { and } 6) ; 7.48(\mathrm{~s}, 1 \mathrm{H}, \text { no. } 8) ; 6.8(\mathrm{~d}, 2 \mathrm{H}, \text { no. } 3 \\
\text { and 5); } 6.29(\mathrm{~d}, 1 \mathrm{H}, \text { no. } 7)\end{array}$ \\
\hline \multirow{3}{*}{ CP-ASA-PCMA } & CP protons: 7.5 (overlap, 1H, no. 2); 7.18 (d, 1H, no. 5); 6.64 (overlap, $1 \mathrm{H}$, no. 8) \\
\hline & $\begin{array}{l}\text { ASA protons: } 7.96(\mathrm{~d}, 1 \mathrm{H}, \text { no. } 5) ; 7.69(\mathrm{~d}, 1 \mathrm{H}, \text { no. } 3) ; 7.61(\mathrm{~d}, 1 \mathrm{H}, \text { no. } 4) ; 6.64 \\
\text { (overlap, } 1 \mathrm{H}, \text { no. } 2)\end{array}$ \\
\hline & $\begin{array}{l}\text { PCMA protons: } 7.5-7.6(\mathrm{~m} \text {, overlap, } 3 \mathrm{H} \text {, no. } 2,6 \text { and } 8) ; 6.75(\mathrm{~d}, 2 \mathrm{H}, \text { no. } 3 \text { and } \\
\text { 5); } 6.3(\mathrm{~d}, 1 \mathrm{H}, \text { no. } 7)\end{array}$ \\
\hline \multirow{2}{*}{ CP-BA } & CP protons: 8.67 (s, 1H, no. 2); 7.94 (overlap, $1 \mathrm{H}$, no. 5); 7.59 (d, 1H, no. 8) \\
\hline & BA protons: $7.94(\mathrm{~d}, 2 \mathrm{H}$, no. 1 and 6$) ; 7.55(\mathrm{~d}, 1 \mathrm{H}$, no. 4$) ; 7.48(\mathrm{~d}, 2 \mathrm{H}$, no. 3 and 5$)$ \\
\hline \multirow{3}{*}{ CP-BA-PCMA } & CP protons: 8.65 (s, 1H, no. 2); 7.94 (d, 1H, no. 5); 7.5 (overlap, 1H, no. 8) \\
\hline & BA protons: $7.94(\mathrm{~d}, 2 \mathrm{H}$, no. 2 and 6$) ; 7.5$ (m, 3H, no. 3,4 and 5) \\
\hline & $\begin{array}{l}\text { PCMA protons: } 7.51(\mathrm{~d}, 3 \mathrm{H}, \text { no. } 2,6 \text { and } 8) ; 6.75(\mathrm{~d}, 2 \mathrm{H}, \text { no. } 3 \text { and } 5) ; 6.29(\mathrm{~d} \text {, } \\
1 \mathrm{H}, \text { no. } 7)\end{array}$ \\
\hline \multirow{2}{*}{ CP-PASA } & CP protons: 8.65 (s, 1H, no. 2); 7.94 (d, 1H, no. 5); 7.59 (d, 1H, no. 8) \\
\hline & PASA protons: $7.35(\mathrm{~d}, 1 \mathrm{H}$, no. 6$) ; 5.98(\mathrm{~d}, 1 \mathrm{H}$, no. 5$) ; 5.9(\mathrm{~s}, 1 \mathrm{H}$, no. 3$)$ \\
\hline \multirow{3}{*}{ CP-PASA-PCMA } & CP protons: 8.67 (s, $1 \mathrm{H}$, no. 2); 7.9 (d, $1 \mathrm{H}$, no. 5); 7.57 (d, 1H, no. 8) \\
\hline & PASA protons: 7.38 (d, 1H, no. 6); 5.97 (d, 1H, no. 5); 5.88 (s, 1H, no. 3) \\
\hline & PCMA protons: $7.1(\mathrm{~d}, 3 \mathrm{H}$, no. 2, 6 and 8$) ; 7.4(\mathrm{~s}, 1 \mathrm{H}), 6.8(\mathrm{~d}, 2 \mathrm{H}$, no. 7$)$ \\
\hline
\end{tabular}

ASA - acetylsalicylic acid, BA - benzoic acid, CP - ciprofloxacin, PASA - p-aminosalicylic acid, PCMA - $p$-coumaric acid

appeared as dd at highest $\delta$ (ppm) which corresponds to the signal at $7.92 \mathrm{ppm}$ in the spectrum of CP-ASA. It is noteworthy that each proton of ASA appeared at two close frequencies with similar multiplicities. According to relative peak areas for protons of ASA and those for $\mathrm{CP}$ it could be concluded that four molecules of ASA were bound to one molecule of $\mathrm{CP}$, albeit with an asymmetrical manner (hence the duplication of proton signals in NMR). None of the protons of $\mathrm{CP}$ appeared to be significantly shifted, but that for proton number 2 became a doublet instead of a singlet. A structural arrangement that fits the NMR data can be proposed. Two molecules of ASA seem to bind to CP through dimer formation with the carboxyl group of $\mathrm{CP}$, therefore minimum or no shift is anticipated for their aromatic protons in the NMR spectrum. The other two molecules, however, are likely to be aligned with the fluorinated ring of $\mathrm{CP}$ in a manner that allows protons 3 and 4 of ASA to lie close to the aromatic ring of $\mathrm{CP}$, leading to the observed shifts. At the same time, such an arrangement would allow for acid-base interaction between the carboxyl of ASA and the basic amino group of piperazine ring. 
For the spectrum of CP-PCMA (Fig. 4c), the characteristic peaks for protons number 5 and 8 of $\mathrm{CP}$ appeared clearly, though with dramatic upfield shifts. The peak for proton number 2 of $\mathrm{CP}$ appeared to completely overlap with that for the characteristic mixed peak for PCMA (at $7.5 \mathrm{ppm}$ ). The integrated peak area ratios most likely accord with two molecules of PCMA bound to one molecule of CP. The observation that the protons of CP were significantly shifted to a lower frequency (more shielded) suggests that each of the aromatic rings of $\mathrm{CP}$ is aligned at some angle with the aromatic ring of PCMA (one molecule of PCMA for each CP ring) so that the aromatic protons of CP would lie in the middle of the aromatic ring.

For the product CP-ASA-PCMA (Fig. 4d), the formation of the ternary molecular structure is evidenced by the characteristic peaks for the protons of CP, ASA and PCMA. The pattern for ASA protons was significantly different from that observed for CP-ASA (binary salt) in the sense that only one signal for each proton on ASA was observed, while in ASAPCMA two peaks were observed for each proton reflecting different molecular arrangement. While some of the ASA protons were shifted to a lower frequency, protons of PCMA and $\mathrm{CP}$ did not show significant shifts. However, according to integrated peak area ratios of properly resolved protons signals, the estimated association ratio between the three compounds accord with 1:1:2 (CP:ASA:PCMA).

For CP-BA (Fig. S1), the protons of both components were observed with no significant shifts in their resonances. Integrated peak area ratios suggested a ratio of association of 1:1 between the two. The ternary product CP-BA-PCMA was also shown to form as the protons of the three original compounds were evident in the NMR spectrum and without significant shifts of resonances. Peak area calculations suggested the formation of a 1:1:1 ternary molecular association product, most likely one of the acidic moieties through the acid-base association with the amino group of $\mathrm{CP}$ and the other molecule as a carboxylic acid dimer with the carboxylic acid of $\mathrm{CP}$.

The NMR spectrum of CP-PASA (Fig. S1) was simple and straightforward with minimum overlapping of signals. All protons of both compounds were shown in the spectrum with a clear association ratio of the two equal to 1:1. The ternary product involving PASA was also evident with an obvious binding ratio of 1:1:1 with minimum shifts in the protons of CP and PASA. However, significant shifts for some protons of CPMA could be observed, i.e., proton No. 7 which appeared to become more shielded upon the ternary structure formation. Overall, NMR spectra provided clear evidence on the formation of binary and ternary salts as products that was in accordance with the results of HPLC ratio analysis.

FTIR spectra for $\mathrm{CP}-\mathrm{HCl}$, each anionic component as well as the products were obtained (Fig. 5). FTIR spectra for the binary products (CP plus any of the employed anions), and ternary products (CP plus PCMA, plus one of the remaining anions) showed clear differences from each of free $\mathrm{CP}$ and/or individual anion (Fig. 5). A summary of the major functional groups' peaks for $\mathrm{CP}$ as well as each of the employed anions and the combination products are presented in Table III.

The most prominent absorption bands of $\mathrm{CP}$ were shown at 3540 (mainly N-H stretching), 1710 ( $\mathrm{C}=\mathrm{O}$, carboxylic), $1620\left(\mathrm{C}=\mathrm{O}\right.$, ketone or $\mathrm{N}-\mathrm{H}$ functional groups $\mathrm{cm}^{-1}$ which accords well with the previous reports (21-24). Reflections for the dimeric carboxyl O-H stretch, and the protonated amino group are seen as multiple bands in the range of 2500$2700 \mathrm{~cm}^{-1}(21-24)$. 

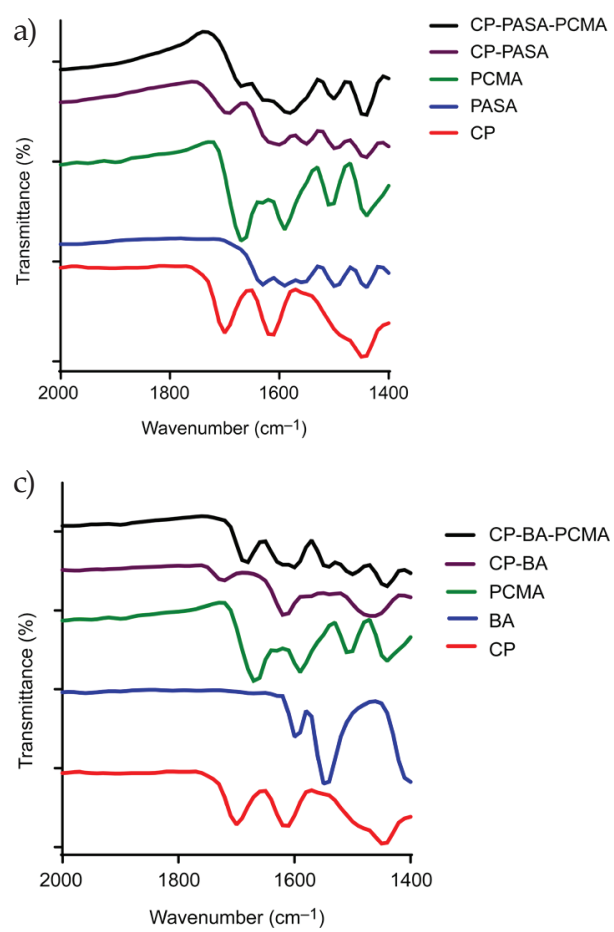
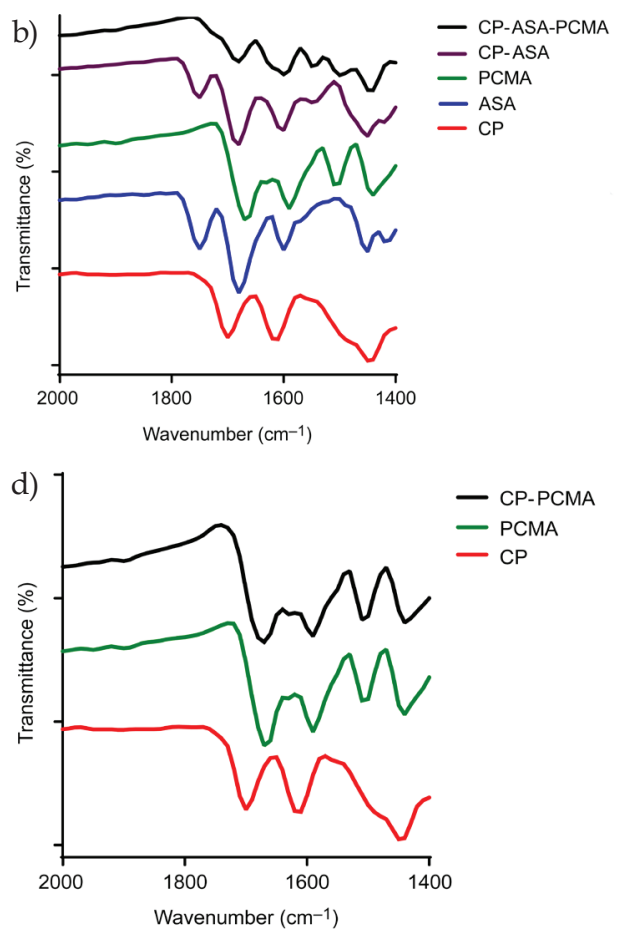

Fig. 5. FTIR spectra (double bond region, $1400-2000 \mathrm{~cm}^{-1}$ ) of the binary and ternary salts obtained with CP and: a) PASA, b) ASA, c) BA, d) PCMA.

Table III. Major functional groups peaks in FTIR spectra of CP, organic anions and their products

\begin{tabular}{llllll}
\hline \multirow{2}{*}{ Compound } & \multicolumn{5}{l}{ Main functional groups, wavenumber $\left(\mathrm{cm}^{-1}\right)$} \\
\cline { 2 - 6 } & $\mathrm{N}-\mathrm{H}$ & $\mathrm{O}-\mathrm{H}($ phenol$)$ & $\mathrm{O}-\mathrm{H}(\mathrm{acid})$ & $\mathrm{C}=\mathrm{O}$ & $\begin{array}{l}\text { Quinolone } \\
\text { carbonyl }(\mathrm{C}=\mathrm{O})\end{array}$ \\
\hline CP & $3530-3400$ & - & $2470-2700$ & 1708 & 1620 \\
BA & - & - & - & 1608 & - \\
PCMA & - & $3375(\mathrm{~b})$ & $2600-3375(\mathrm{~b})$ & 1670 & - \\
CP-BA & 3425 & - & - & $1731(\mathrm{w})$ & $1621(\mathrm{~s})$ \\
CP-PCMA & $3389(\mathrm{~m}, \mathrm{~b})$ & - & $2600-3389$ & $1676(\mathrm{~s})$ & 1654 \\
CP-BA-PCMA & - & - & - & 1689 & 1631,1629 \\
ASA & - & $2896(\mathrm{~m}, \mathrm{~b})$ & - & $1753(\mathrm{~m}), 1683(\mathrm{~s})-$ \\
CP-ASA & - & $2854(\mathrm{~m}, \mathrm{~b})$ & - & $1753(\mathrm{w}), 1687(\mathrm{~s})-$ \\
CP-ASA-PCMA & - & $3037(\mathrm{~m}, \mathrm{~b})$ & $2400-3037(\mathrm{~m})$ & $1691(\mathrm{~m})$ & $1630(\mathrm{~m})$ \\
PASA & - & $2800-3500(\mathrm{w}, \mathrm{b})$ & & 1627 & - \\
CP-PASA & $3414(\mathrm{w}, \mathrm{b})$ & - & $2400-3414(\mathrm{w}, \mathrm{b})$ & $1704(\mathrm{~m})$ & $1629(\mathrm{~s}), 1621(\mathrm{~s})$ \\
CP-PASA-PCMA & - & $3460(\mathrm{~m}, \mathrm{~b})$ & $2400-3460(\mathrm{w}, \mathrm{b})$ & 1674 & 1635 \\
\hline
\end{tabular}

ASA - acetylsalicylic acid, BA - benzoic acid, CP-ciprofloxacin, PASA - $p$-aminosalicylic acid, PCMA - $p$-coumaric acid $\mathrm{b}$ - broad, $\mathrm{m}$ - medium, $\mathrm{s}$ - strong, $\mathrm{w}$ - weak 
Of the most obvious changes in the IR spectra were the changes noticed in $>3100 \mathrm{~cm}^{-1}$ regions, which is mainly assigned to the exchangeable protons $\mathrm{OH}$ and $\mathrm{NH}$ stretching. When compared to $\mathrm{CP}$, peaks in such regions became generally broader and less intense upon the formation of the product suggesting stronger hydrogen bond structure. The characteristic absorption band for carboxylic carbonyl at $1708 \mathrm{~cm}^{-1}$ disappeared completely or shifted to a lower frequency for most of the salts of CP and with employed organic acids, either as binary or ternary products (Fig. 5 and Table III). However, the magnitude of such changes varied between products with the minimum being observed for the binary salt involving CP and PCMA (Fig. 5d). The characteristic carbonyl absorption bands for each organic acid (1670-1750 $\left.\mathrm{cm}^{-1}\right)$ were observed in the relevant binary products (CP-organic anion) with some minor shifts. However, in ternary products involving CP, PCMA and one of the other organic acids, only the characteristic absorption band relevant to the carbonyl group of PCMA was observable while that of the other anion disappeared. Thus one might think that in these cases the actual product simply consists of only CP and PCMA while the other added anion remained in solution and did not participate in the formation of the product. However, the overall spectra for binary and ternary products were obviously different (Fig. 5). Additionally, NMR data showed unequivocally the presence of the three

a)

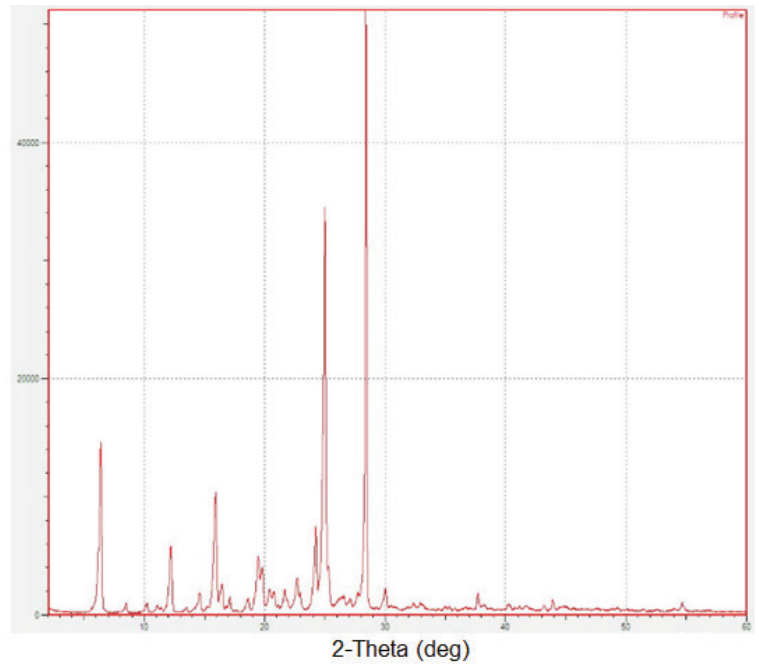

b)

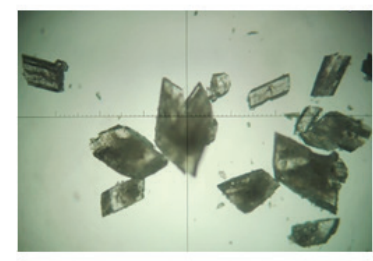

c)

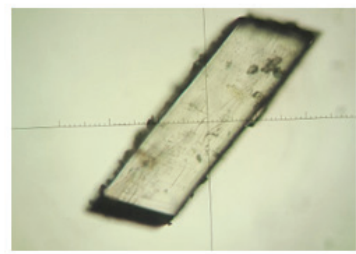

d)

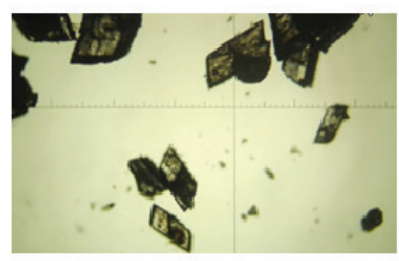

Fig. 6. a) X-ray diffraction pattern of CP-PASA. b), c) and d) are microscopic images of CP-PASA, CP-BAPCMA, and CP-BA, resp. 
components in cases of ternary products (CP, PCMA and other organic anions). Although binary salts of $\mathrm{CP}$ with carboxylic acids have been frequently reported, to the best of our knowledge the tri-molecular salts are reported for the first time in this study.

$X$-ray diffraction. - X-ray diffraction patterns were obtained for some of the crystalline products (CP-BA-PCMA, Fig. 6a) along with microscopic images for the crystals of CP-PASA, CP-BA-PCMA, and CP-BA (Figs. 6b-d). The crystalline nature of the products and the obtained $\mathrm{X}$-ray diffraction pattern reflect a good level of purity due to the existence of very sharp and relatively few peaks, which enhances the confidence in the homogeneity and thus the purity of the products.

Antimicrobial activity. - A summary of the obtained MIC values for the prepared salts is presented in Table III. Ciprofloxacin $(\mathrm{CP}-\mathrm{HCl})$ was used as a reference compound.

Based on the presented results (Table IV) none of the individual four anions exhibited interesting antibacterial activity against the two examined strains, Staphylococcus aureus and Staphylococcus epidermidis. The results for CP were generally comparable to values previously reported $(25,26)$. CP showed four times higher antimicrobial activity against $S$. epidermidis compared to $S$. aureus. Different products showed different activities towards S. aureus and S. epidermidis. CP-BA exhibited higher activity against $S$. aureus while CP-BA-PCMA, CP-ASA, CP-ASA-PCMA, and CP-PASA were more active against S. epidermidis. Most of the salt products (5 out of 7) had either comparable or higher antimicrobial activity than $\mathrm{CP}$ against both strains. The most active compounds against S. epidermidis and $S$. aureus were CP-PASA $\left(M I C=1 \mu \mathrm{mol} \mathrm{L}{ }^{-1}\right)$ and CP-PCMA $\left(M I C=5 \mu \mathrm{mol} \mathrm{L}^{-1}\right)$, resp., which were about fourteen times more potent than native CP. Our results confirm that the prepared $\mathrm{CP}$-salts modulate the antimicrobial activity of $\mathrm{CP}$, favorably, in most cases. The observed improvement in their antimicrobial effect compared to $\mathrm{CP}$ might be due to their

Table IV. Antimicrobial activity (MIC) for the examined compounds

\begin{tabular}{lcc}
\hline Compound & $\begin{array}{c}\text { MIC (Staphylococcus epidermidis) } \\
\left(\mu \mathrm{mol} \mathrm{L}^{-1}\right)^{\mathrm{a}}\end{array}$ & $\begin{array}{c}\text { MIC (Staphylococcus aureus) } \\
\left(\mu \mathrm{mol} \mathrm{L}^{-1}\right)^{\mathrm{a}}\end{array}$ \\
\hline Ciprofloxacin $(\mathrm{CP}-\mathrm{HCl})$ & 17 & 68 \\
BA & $>1639$ & $>1639$ \\
PCMA & 1219 & 1219 \\
ASA & $>1111$ & $>111$ \\
PASA & $>1307$ & $>1307$ \\
CP-BA & 11 & 5 \\
CP-PCMA & 5 & 5 \\
CP-BA-PCMA & 10 & 20 \\
CP-ASA & 5 & 18 \\
CP-ASA-PCMA & 19 & 37 \\
CP-PASA & 1 & 10 \\
CP-PASA-PCMA & 39 & 39 \\
\hline
\end{tabular}

CP - ciprofloxacin, ASA - acetylsalicylic acid, BA - benzoic acid, PASA - p-aminosalicylic acid, PCMA - p-coumaric acid

${ }^{a}$ Mean values from triplicate measurements repeated twice for each bacterium and each tested compound. 

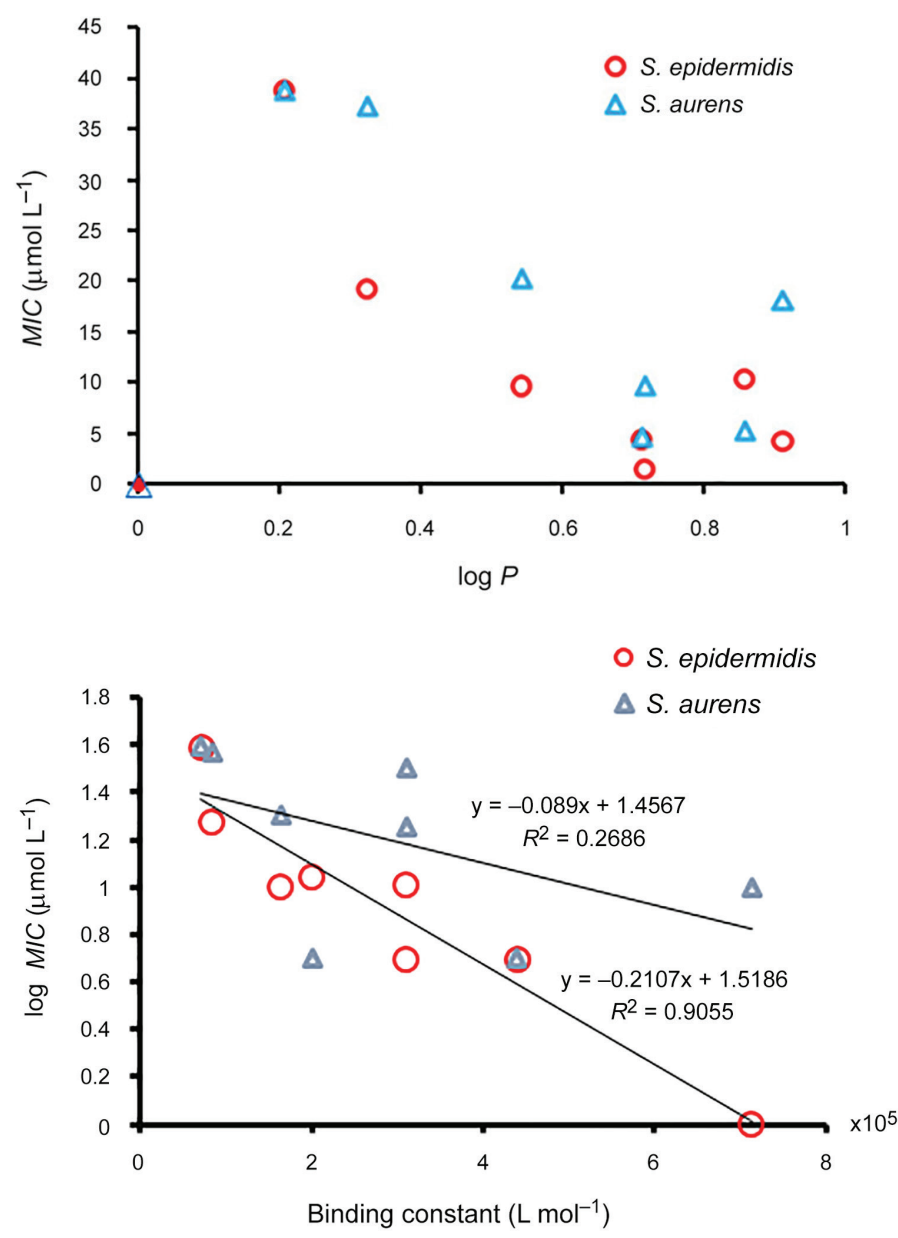

Fig. 7. a) A plot of $M I C$ values of different products between their measured $\log P$ values. b) A plot of MIC between the binding constants of the products. Each point in the plot represents a different product.

increased lipophilicity which in turn expectedly enhances permeation through the cell membrane with consequent enhancement of antimicrobial activity. Another possible explanation might include intact ion pair which interacts with some important cellular targets in a different way.

In order to get further insights, a plot between the obtained MIC values and the experimentally measured $\log P$ values was established (Fig. 7a). Interestingly, Fig. 7a shows that, with both $S$. aureus and S. epidermidis, there was an optimum value of $\log P$ that provided the best antimicrobial activity. The value was almost the same with both strains and was around 0.75. Our finding is consistent with the previous work of Vázquez et al. (27) 
who studied the relationship between the antimicrobial activity of quinolones and their partition coefficient values and reported similar tendencies and the existence of an optimum value of $\log P$ for maximum antimicrobial activity against $E$. coli. In their study, the optimum value of the partition coefficient was $\sim 2.5$ which is equivalent to $\log P$ of 0.4 . However, in their study, the optimization of partition coefficient values of quinolone compounds was only achievable through the synthesis of new derivatives of $\mathrm{CP}$ having covalently attached side chains of various lengths of (27). In our study, we demonstrate the possibility of achieving optimum $\log P$ of $\mathrm{CP}$ derivatives simply through the proper choice of the counteranion to form ion pair (salt).

Binding of CP salts to DNA. - CP had been shown to bind to DNA influencing its mode of antimicrobial action $(28,29)$. Therefore, we decided to study the ability of the various prepared salts to interact with calf thymus DNA (CT DNA). UV/VIS spectroscopic titration of samples of CP with CT DNA revealed a progressive spectral shift at $\sim 323 \mathrm{~nm}$ as more of DNA was added (Fig. S2). DNA itself shows no absorption at $323 \mathrm{~nm}$, therefore, the observed shift made us inclined to believe of drug-DNA complex formation and called for estimation of the respective binding isotherm and binding constant.

DNA-drug binding constants obtained for the various derivatives of $\mathrm{CP}$ revealed almost one order of magnitude between the largest $\left(7.1 \times 10^{5} \mathrm{~L} \mathrm{~mol}^{-1}\right)$ and the weakest binding derivatives $\left(8.3 \times 10^{4} \mathrm{~L} \mathrm{~mol}^{-1}\right)($ Fig. $7 \mathrm{~b})$. This prompted us to search for a potential correlation between the binding constant and the observed $M I C$ values against the two tested microorganisms. When $\log$ MIC values for each $\mathrm{CP}$ salt were plotted against their corresponding DNA binding constants, a reasonable linear correlation was shown with a correlation coefficient of 0.90 for $S$. epidermidis but there was no reasonable correlation for $S$. aureus (Fig. $7 \mathrm{~b}$ ). The strong correlation noticed herein between the DNA binding constant of the CP salts and their observed MIC values against $S$. epidermidis supports the previous reports (30) of the potential importance of DNA binding ability of quinolones in their mechanism of action. Although the mechanism of action for quinolones is generally accepted as DNA gyrase inhibitors, some reports have demonstrated a direct correlation between DNA binding constant and the measured antimicrobial activity (31). This is in line with our findings where a clearly strong correlation was noticed with S. epidermidis. The provided data might suggest that the prepared salts remained as intact ion pairs even inside the microbial cells.

\section{CONCLUSIONS}

Novel binary and ternary salts of $\mathrm{CP}$ were prepared using four different organic anions. The counteranions played an important role in dictating important physical, thermal and antimicrobial properties of CP. The prepared salts have been adequately characterized and evaluated for their antimicrobial activities. Our findings have shown that the prepared salts of $\mathrm{CP}$ are maintained at very low concentrations sufficient to affect bacterial cells. The prepared derivatives have improved physicochemical properties compared to parent $\mathrm{CP}$ and exhibited a range of lipophilicities that resulted in differences in their antimicrobial activities. Therefore, the products were shown able to modulate the antimicrobial activity of the parent $\mathrm{CP}$. 
Our results suggested that lipophilicity might correlate with antimicrobial activity and showed that an optimum value of lipophilicity seemed to be necessary to maximize the antimicrobial activity. While the main influence of lipophilicity appears to be by improving the permeation through the cell membrane, our study has suggested that the intact salt structure is likely to get into the cell and, thus, might interact with the bacterial DNA leading to differences in the antimicrobial activity in a manner proportional to the extent of DNA binding. The improved antibacterial activity of the prepared salts is of particular interest, especially in topical and ophthalmic preparations.

Acknowledgements. - The authors wish to thank the Deanship of Scientific Research at The University of Jordan for financial support (Grant recommendation No. 27/2014-2015).

Supplementary material available upon request.

\section{REFERENCES}

1. D. M. Campoli-Richards, J. P. Monk, A. Price, P. Benfield, P. A. Todd and A. Ward, Ciprofloxacin. A review of its antibacterial activity, pharmacokinetic properties and therapeutic use, Drugs 35 (1988) 373-447; https://doi.org/10.2165/00003495-198835040-00003

2. A. D. Pranger, J. W. C. Alffenaar and R. E. Aarnoutse, Fluoroquinolones, the cornerstone of treatment of drug-resistant tuberculosis: A pharmacokinetic and pharmacodynamics approach, Curr. Pharm. Des. 17 (2011) 2900-2930; https://doi.org/10.2174/138161211797470200

3. L. D. Ross and C. M. Riley, Aqueous solubilities of some variously substituted quinolone antimicrobials, Int. J. Pharm. 63 (1990) 237-250; https://doi.org/10.1016/0378-5173(90)90130-V

4. A. O. Surov, A. N. Manin, A. P. Voronin, K. V. Drozd, A. A. Simagina, A. V. Churakov and G. L. Perlovich, Pharmaceutical salts of ciprofloxacin with dicarboxylic acids, Eur. J. Pharm. Sci. 77 (2015) 112-121; https://doi.org/10.1016/j.ejps.2015.06.004

5. M. E. Olivera, R. H. Manzo, H. E. Junginger, K. K. Midha, V. P. Shah, S. Stavchansky, J. B. Dressman and D. M. Barends, Biowaiver monographs for immediate release solid oral dosage forms: ciprofloxacin hydrochloride, J Pharm Sci. 100 (2011) 22-33; https://doi.org/10.1002/jps.22259

6. H. Arakawa, Y. Shirasaka and M. Haga, Active intestinal absorption of fluoroquinolone antibacterial agent ciprofloxacin by organic anion transporting polypeptide, Biopharm. Drug Dispos. 33 (2012) 332-341; https://doi.org/10.1002/bdd.1809

7. Z. Iqbal, A. Khan, A. Naz, J. Khan and G. Khan, Pharmacokinetic interaction of ciprofloxacin with diclofenac: a single-dose, two-period crossover study in healthy adult volunteers, Clin. Drug Invest. 29 (2009) 275-281; https://doi.org/10.2165/00044011-200929040-00006

8. D. El-Sabawi, R. Abu-Dahab, A. G. Al Bakri and I. I. Hamdan, Studies on the interaction between ciprofloxacin hydrochloride and diclofenac sodium, Trop. J. Pharm. Res. 18 (2019) 377-384; https:// doi.org/10.4314/tjpr.v18i2.22

9. P. P. Bag, S. Ghosh, H. Khan, R. Devarapalliand and C. M. Reddy, Drug-drug salt forms of ciprofloxacin with diflunisal and indoprofen, Cryst. Eng. Commun. 16 (2014) 7393-7396; https://doi. org/10.1039/C4CE00631C

10. C. Florindo, A. Costa, C. Matos, S. L. Nunes, A. N. Matias, C. M. Duarte, L. P. Rebelo, L. C. Branco and I. M. Marrucho, Novel organic salts based on fluoroquinolone drugs: synthesis, bioavailability and toxicological profiles, Int. J. Pharm. 469 (2014) 179-189; https://doi.org/10.1016/j. ijpharm.2014.04.034

11. M. Ali and M. E. Byrne, Challenges and solutions in topical ocular drug-delivery systems, Expert. Rev. Clin. Pharmacol. 1 (2008) 145-161; https://doi.org/10.1586/17512433.1.1.145 
12. A. A. Firsov, I. Y. Lubenko, M. V. Smirnova, E. N. Strukova and S. H. Zinner, Enrichment of fluoroquinolone-resistant Staphylococcus aureus: oscillating ciprofloxacin concentrations simulated at the upper and lower portions of the mutant selection window, J. Antimicrob. Chemother. 52 (2008) 1924-1928; https://doi.org/10.1128/AAC.01371-07

13. N. A. Lozano-Huntelman, N. Singh, A. Valencia, P. Mira, M. Sakayan, I. Boucher, S. Tang, K. Brennan, C. Gianvecchio and S. Fitz-Gibbon, Evolution of antibiotic cross-resistance and collateral sensitivity in Staphylococcus epidermidis using the mutant prevention concentration and the mutant selection window, Evol. Appl. 13 (2020) 808-823; https://doi.org/10.1111/eva.12903

14. A. Espinel-Ingroff, A. Fothergill, M. Ghannoum, E. Manavathu, L. Ostrosky-Zeichner and M. Pfaller, Quality control and reference guidelines for CLSI broth microdilution method (m38-a document) for susceptibility testing of anidulafungin against molds, J. Clin. Microbiol. 45 (2007) 2180-2182; https://doi.org/10.1128/JCM.00399-07

15. A. Wolfe, G. H. Shimer and J. T. Meehan, Polycyclic aromatic hydrocarbons physically intercalate into duplex regions of denatured DNA, Biochemistry 26 (1987) 6392-6396; https://doi.org/10.1021/ bi00394a013

16. P. Gould, Salt selection for basic drugs, Int. J. Pharm. 33 (1986) 201-217; https://doi.org/10.1016/03785173(86)90055-4

17. K. Zhang, L. Dai and N. Chetwyn, Simultaneous determination of positive and negative pharmaceutical counter ions using mixed-mode chromatography coupled with charged aerosol detector, J. Chromatogr. A 1217 (2010) 5776-5784; https://doi.org/10.1016/j.chroma.2010.07.035

18. S. Bouabdallah, H. Trabelsi, M. R. Driss and S. Touil, Determination and degradation study of enalapril maleate by high performance liquid chromatography, Pharm. Chem. J. 51 (2017) 735-741; https://doi.org/10.1007/s11094-017-1684-2

19. B. W. Pack and D. S. Risley, Evaluation of a monolithic silica column operated in the hydrophilic interaction chromatography mode with evaporative light scattering detection for the separation and detection of counter-ions, J. Chromatogr. A 1073 (2005) 269-275; https://doi.org/10.1016/j.chroma.2004.09.061

20. M. Takač, Effects of substituents on the NMR features of basic bicyclic ring systems of fluoroquinolone antibiotics and the relationships between NMR chemical shifts, molecular descriptors and drug-likeness parameters, Acta Pharm. 60 (2010) 237-254; https://doi.org/10.2478/v10007-010-0023-x

21. S. Durgapal, S. Mukhopadhyay and L. Goswami, Preparation, characterization and evaluation of floating microparticles of ciprofloxacin, Int. J. Appl. Pharm. 9 (2017) 1-8; https://doi.org/10.22159/ ijap.2017v9i1.14183

22. H. Changa, W. T. Jianga, Z. Lib, C. Y. Kuoc, Q. Wud, J. S. Jeana and G. Lvea, Interaction of ciprofloxacin and probe compounds with palygorskite PFl-1, J. Hazard. Mat. 303 (2016) 55-63; https:// doi.org/10.1016/j.jhazmat.2015.10.012

23. M. P. López-Gresa, R. Ortiz and L. Perelló, Interactions of metal ions with two quinolone antimicrobial agents (cinoxacin and ciprofloxacin). Spectroscopic and X-ray structural characterization. Antibacterial studies, J. Inorg. Biochem. 92 (2002) 65-74; https://doi.org/10.1016/S0162-0134(02)004877

24. K. Karimi, E.Pallagi, P. Szabó-Révész, I. Csóka and R. Ambrus, Development of a microparticlebased dry powder inhalation formulation of ciprofloxacin hydrochloride applying the quality by design approach, Drug Des. Dev. Ther. 10 (2016) 3331-3343; https://doi.org/10.2147/DDDT.S116443

25. J. G. Holler, S. B. Christensen, H. C. Slotved, H. B. Rasmussen, A. Gúzman, C. E. Olsen, B. Petersen and P. Mølgaard, Novel inhibitory activity of the Staphylococcus aureus NorA efflux pump by a kaempferol rhamnoside isolated from Persea lingue Nees, J. Antimicrob. Chemother. 67 (2012) 1138-1144; https://doi.org/10.1093/jac/dks005 
26. G. P. Gesu, F. Marchetti, L. Piccoli and A. Cavallero, Levofloxacin and ciprofloxacin in vitro activities against 4,003 clinical bacterial isolates collected in 24 Italian laboratories, J. Antimicrob. Chemother. 47 (2003) 816-819; https://doi.org/10.1128/aac.47.2.816-819.2003

27. J. Vázquez, S. Merino, Ò. Doměnech, M. Berlanga, M. Viñas and M. Montero, Determination of the partition coefficients of a homologous series of ciprofloxacin: influence of the N-4 piperazinyl alkylation on the antimicrobial activity, Int. J. Pharm. 220 (2001) 53-62; https://doi.org/10.1016/ s0378-5173(01)00646-9

28. I. Vilfan, P. Drevenšek, I. Turel and U. N. Poklar, Characterization of ciprofloxacin binding to the linear single- and double-stranded DNA, Biochim. Biophys. Acta 1628 (2003) 111-122; https://doi. org/10.1016/s0167-4781(03)00135-0

29. D. Hooper, Mode of action of fluoroquinolones, Drugs 58 (1999) 6-10; https://doi.org/10.2165/00003495199958002-00002

30. K. J. Aldred, R. J. Kerns and N. Osheroff, Mechanism of quinolone action and resistance, Biochemistry 53 (2014) 1565-1574;https://doi.org/10.1021/bi5000564

31. C. Noble, F. Barnard and A. Maxwell, Quinolone-DNA interaction: sequence-dependent binding to single-stranded DNA reflects the interaction within the gyrase-DNA complex, Antimicrob. Agents Chemother. 47 (2003) 854-862; https://doi.org/10.1128/aac.47.3.854-862.2003 